\title{
EXCAVATION DATA AND FAILURE INVESTIGATION ALONG TUNNEL OF SYMBOL MOUNTAIN
}

\author{
Chatziangelou M., Thomopoulos Ach., Christaras B. \\ Department of Geology, Aristotle University of Thessaloniki, Greece, mcha@ geo.auth.gr, \\ christar@geo.auth.gr
}

\begin{abstract}
The tunnel of Symbol Mountain, which is $1160 \mathrm{~m}$ long, is placed on South-west of Kavala City at Northern Greece. The tunnel consists of two bores with NW-SE direction, which are connected with two small tunnels. The stability of the rock mass was limited, during the excavation, because the rock mass was often changing, the faults are open, and the aquifer is placed above the excavation.

The aim of the present paper is to describe the dangerous geological status of Symbol Mountain and to propose excavation solutions of the unexpected failure conditions.

For the above reasons, the sudden changes of the rock mass quality along the tunnel excavation are described. The causes of the geological failures were investigated and the failures were classified. Furthermore, the efficacy of support measures was tested and a relationship between the apparent face of wedges and the shotcrete thickness was proposed.
\end{abstract}

Key words: Tunnels, support measures, wedges, slidings, decollement, anchors, bolts, shotcrete, swellex, excavation.

\section{Introduction}

The tunnel of Symbol Mountain is geotechnical located on Rodope mass. The excavation of the tunnel passed through alternations of gneiss, schists and marbles. The quality of the rock formations often changes from sound to weathered. It is, usually, heavily jointed and in many cases is folded. Furthermore, the presence of chloritic schist, lengthen $400 \mathrm{~m}$, caused numerous unexpected failures and support problems. So, the excavation needed to be extremely careful, and for this reason a combination of excavation methods were used. The presence of an opened vertical fault, which is just placed at the exit of the tunnel and creates a shear zone about $400 \mathrm{~m}$ long, increased the stability problems. The water table is placed above the tunnel.

\section{Rock mass quality}

The beginning of the tunnel, the rock mass consists of fair quality gneiss with pegmatite veins, although there is a part of the tunnel between ch.36+300-ch.36+400 where the quality of a part of gneiss is very poor (Bieniawski, 1989, Hook, 2004). Walking along the tunnel, the rock mass quality becomes poor and or very poor near the schist formation. At the middle of the tunnel (ch. $35+800$ ch.36+300), there is a fair quality lens of marble. Walking to the outlet of the tunnel, we met alternations of gneiss and marble medium and poor qualified. Between ch. 36+500-ch. 36+700, there is a formation of chloritic schistolite of poor quality. That geological formation caused numerous problems during the excavation, as it deformated very quickly after it was excavated. The last part of the tunnel is placed along a shear zone of an opened vertical fault 150/70 F (Fig.1). 


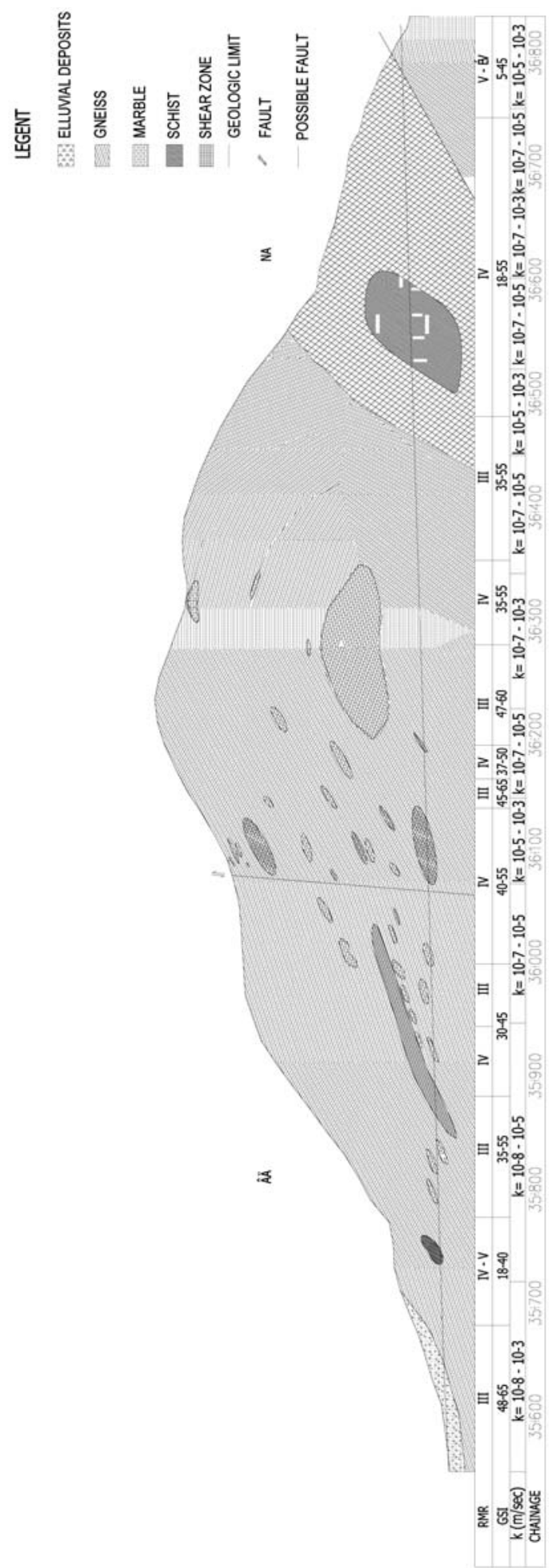

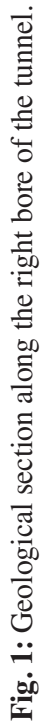




\begin{tabular}{|c|c|c|c|c|c|c|c|c|c|c|c|c|c|c|c|c|c|c|c|c|c|c|c|c|}
\hline$\because$ & & & & & & & & & & & & & & & & $\frac{n}{8}$ & & & & & & & & \\
\hline$\Xi$ & & & $\frac{\infty}{\stackrel{\infty}{i g}}$ & & & & & 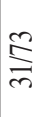 & & $\frac{16}{8}$ & & & & $\begin{array}{l}n \\
\infty \\
i n\end{array}$ & & $\begin{array}{l}n \\
\stackrel{n}{2} \\
\stackrel{2}{5}\end{array}$ & & $\begin{array}{l}\frac{\mathfrak{o}}{2} \\
\stackrel{2}{=}\end{array}$ & & $\begin{array}{l}\sim \\
\sim \\
\stackrel{\sim}{\curvearrowright} \\
\text { D } \\
\end{array}$ & & & & $\frac{n}{\infty}$ \\
\hline 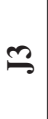 & & & 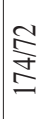 & $\begin{array}{l}n \\
8 \\
8\end{array}$ & $\underset{\sim}{\stackrel{2}{\Im}}$ & 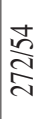 & $\stackrel{\bar{m}}{\partial}$ & $\stackrel{\infty}{\stackrel{i}{二}}$ & $\frac{m}{f}$ & $\begin{array}{l}\sim \\
\infty \\
\text { ऽ } \\
\text { స్ }\end{array}$ & & $\stackrel{\approx}{\stackrel{\sim}{\gamma}}$ & $\underset{\infty}{\mathbb{N}}$ & 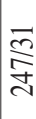 & 守 & $\underset{\sqrt{6}}{\overrightarrow{7}}$ & $\frac{d}{8}$ & $\begin{array}{l}\text { ֶై } \\
\text { ב }\end{array}$ & $\begin{array}{l}\sim \\
a \\
\stackrel{\infty}{a} \\
\stackrel{a}{a}\end{array}$ & 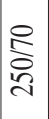 & $\begin{array}{l}\infty \\
\infty \\
\infty \\
\infty \\
\sim\end{array}$ & & $\frac{m}{\stackrel{f}{f}}$ & 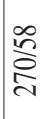 \\
\hline$\stackrel{\Omega}{S}$ & 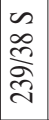 & 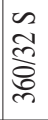 & 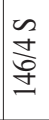 & 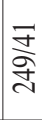 & 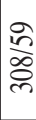 & $\frac{0}{8}$ & $\frac{\sqrt{n}}{2}$ & $\begin{array}{l}\sim \\
\stackrel{a}{a} \\
\infty \\
\approx \\
\approx\end{array}$ & $\begin{array}{l}n \\
\text { on } \\
\text { 学 } \\
\text { m }\end{array}$ & 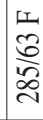 & 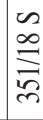 & $\begin{array}{l}\bar{\sigma} \\
\infty \\
\approx \\
\end{array}$ & 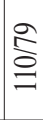 & 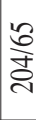 & $\frac{\underset{f}{d}}{\underset{d}{d}}$ & 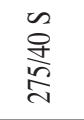 & $\begin{array}{l}\infty \\
\infty \\
\infty \\
\infty \\
\end{array}$ & $\frac{\pi}{\tilde{\sigma}}$ & $\begin{array}{l}\text { 崩 } \\
\text { तु } \\
\text { 官 }\end{array}$ & $\frac{\mathfrak{N}}{\mathscr{O}}$ & $\frac{2}{2}$ & $\frac{8}{2}$ & $\frac{8}{n}$ & $\frac{\stackrel{2}{2}}{2}$ \\
\hline$\Xi$ & $\begin{array}{l}\infty \\
\infty \\
⿱ \infty \\
⿱ 乛 \\
=\end{array}$ & 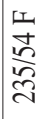 & 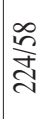 & $\frac{\tilde{0}}{\tilde{n}}$ & $\underset{\substack{\mathrm{g} \\
\mathrm{N}}}{\mathrm{N}}$ & $\underset{\infty}{\infty}$ & $\underset{\sim}{\stackrel{n}{\rho}}$ & $\stackrel{\text { }}{\stackrel{2}{2}}$ & 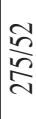 & 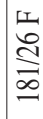 & \begin{tabular}{l}
$\frac{0}{8}$ \\
\multirow{d}{*}{}
\end{tabular} & 总 & $\begin{array}{l}\stackrel{2}{\Sigma} \\
\stackrel{n}{\sim}\end{array}$ & $\stackrel{5}{5}$ & $\begin{array}{l}n \\
8 \\
8 \\
\text { 年 }\end{array}$ & $\stackrel{E}{\equiv}$ & 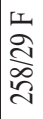 & 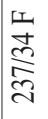 & 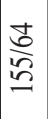 & 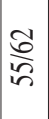 & $\begin{array}{l}\infty \\
0 \\
0 \\
0\end{array}$ & $\mid \begin{array}{l}n \\
0 \\
\frac{n}{n} \\
\frac{n}{n}\end{array}$ & 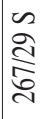 & 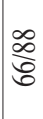 \\
\hline 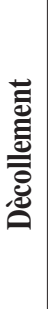 & & & & & & & & & & & & & & $\frac{5}{\underline{\sigma}}$ & & 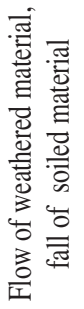 & 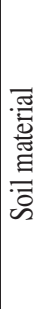 & 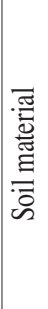 & & 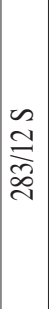 & & 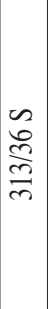 & 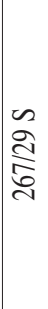 & $\frac{n}{2}$ \\
\hline
\end{tabular}

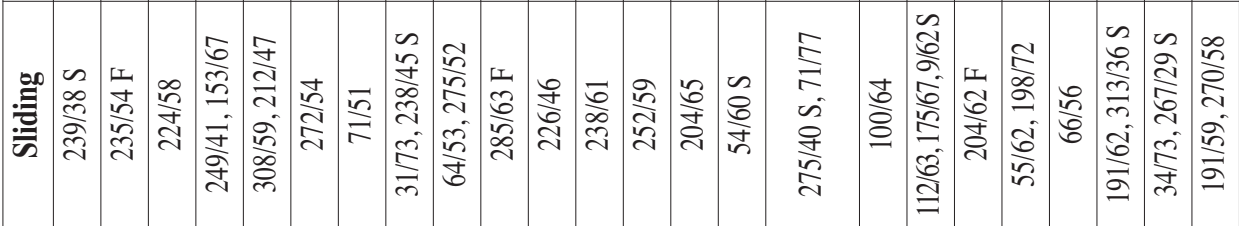

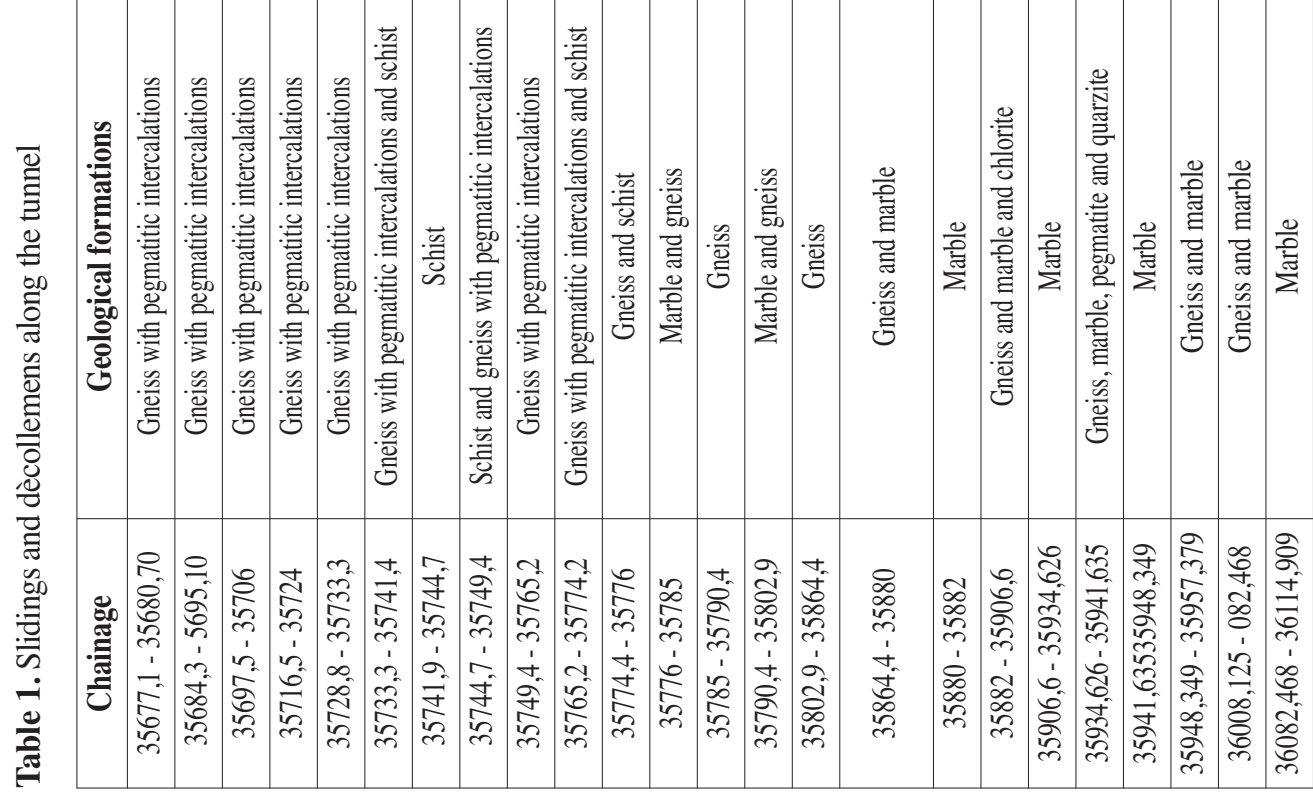




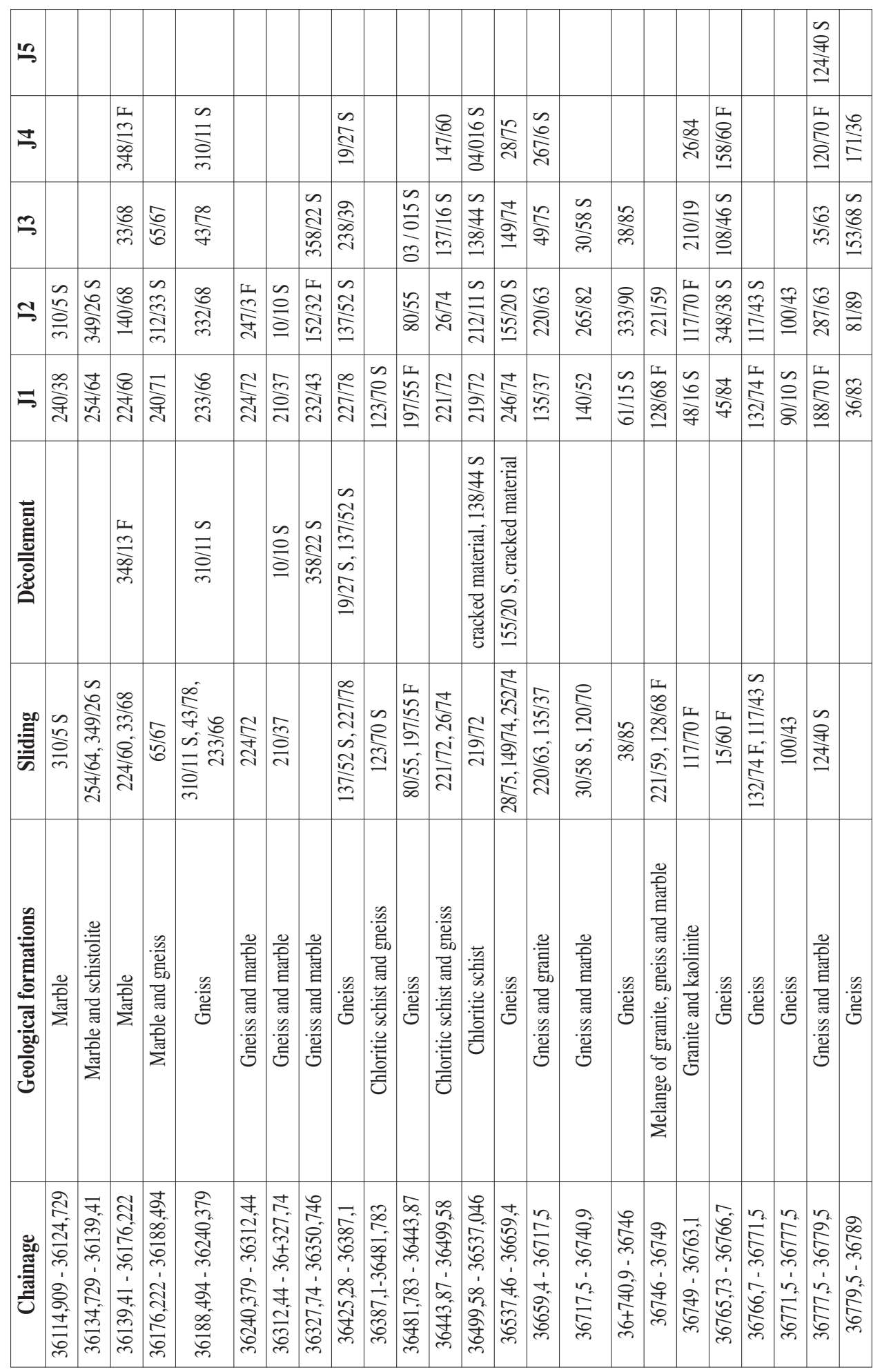




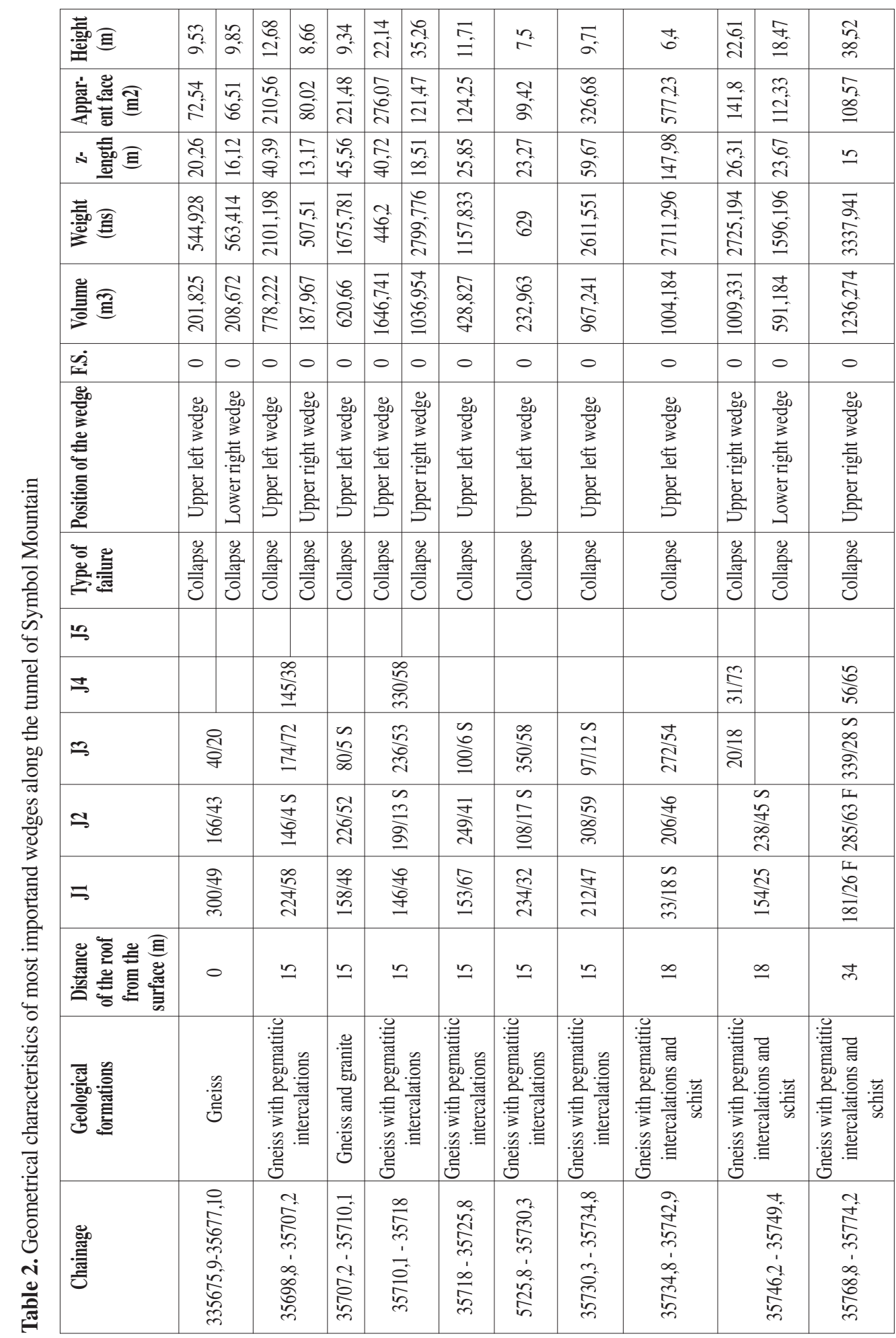




\begin{tabular}{|c|c|c|c|c|c|c|c|c|c|c|c|c|c|c|}
\hline 蒙䔍 & 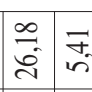 & $\overline{\mathcal{I}}$ & $\vec{F}$ & : & s & 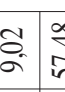 & $\Rightarrow$ & $\stackrel{\text { ga }}{=}$ & $\stackrel{\Xi}{\Xi}$ & 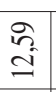 & \begin{tabular}{l|l}
$\stackrel{2}{\circ}$ & $\bar{\gamma}$ \\
$\dot{f}$ &
\end{tabular} & के & 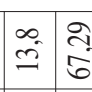 & $\bar{c}$ \\
\hline 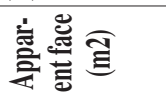 & 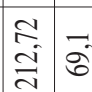 & ঞ̊ & $\begin{array}{l}\overbrace{1} \\
\stackrel{\infty}{=}\end{array}$ & $\begin{array}{l}\infty \\
8 \\
0\end{array}$ & I & 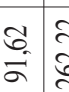 & 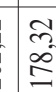 & $\frac{m}{d}$ & 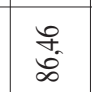 & 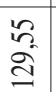 & 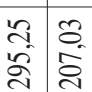 & $\underset{\text { in }}{\text { in }}$ & 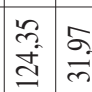 & $\bar{s}$ \\
\hline 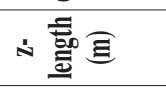 & 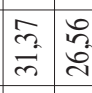 & $\frac{n}{2}$ & 尌 & $\underset{\sigma}{\sigma}$ & 专 & 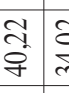 & $\stackrel{\infty}{\rightleftharpoons}$ & $\begin{array}{l}\text { fo } \\
f\end{array}$ & $\begin{array}{l}\vec{n} \\
\text { n }\end{array}$ & लें & 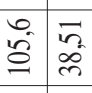 & $\begin{array}{l}\infty \\
\stackrel{ \pm}{ \pm} \\
\end{array}$ & \begin{tabular}{l|l}
$\infty$ & 3 \\
\hdashline & $\infty$ \\
\hdashline & $\infty$
\end{tabular} & $c^{2} \frac{g}{i}$ \\
\hline 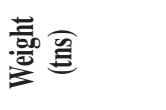 & 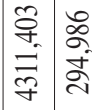 & 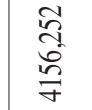 & 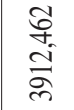 & $\begin{array}{l}\vec{F} \\
\stackrel{7}{a}\end{array}$ & 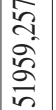 & 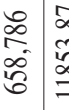 & 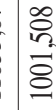 & . & 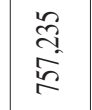 & 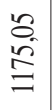 & 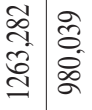 & â & 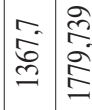 & 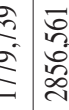 \\
\hline 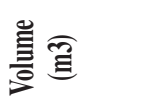 & 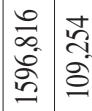 & 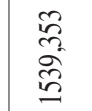 & $\frac{\bar{y}}{2}$ & 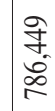 & $y$ & 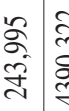 & $\begin{array}{l}\text { בి } \\
\text { Dे } \\
\text { - }\end{array}$ & 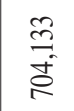 & 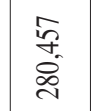 & 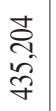 & 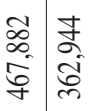 & 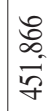 & $\begin{array}{ll}0 \\
0 \\
0 \\
0 \\
0 \\
0\end{array}$ & : \\
\hline 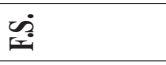 & 00 & 0 & 0 & o & 0 & 00 & 0 & 0 & 0 & 0 & 00 & 0 & 00 & 00 \\
\hline 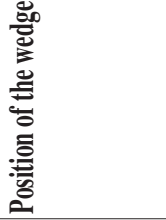 & 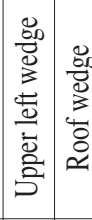 & 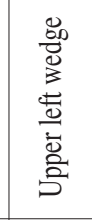 & 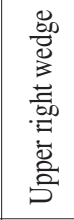 & 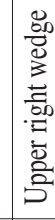 & $\mid$ & 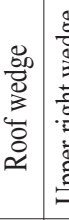 & 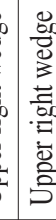 & 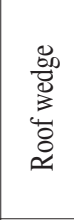 & 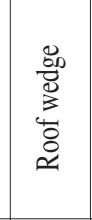 & 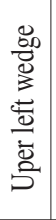 & 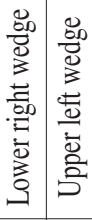 & 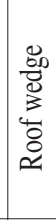 & 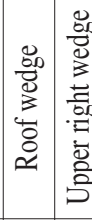 & 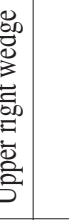 \\
\hline 言善 & 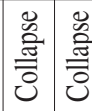 & 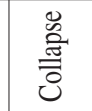 & $\begin{array}{l}\frac{0}{2} \\
\frac{2}{3} \\
\end{array}$ & $\mid \begin{array}{l}\frac{0}{3} \\
\frac{3}{3}\end{array}$ & 童 & 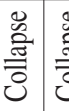 & 这 & 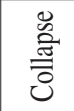 & 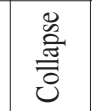 & 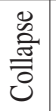 & 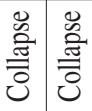 & 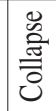 & 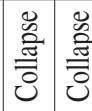 & 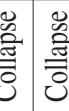 \\
\hline is & & & & & & & & & & & & 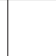 & 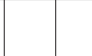 & \\
\hline 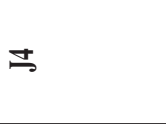 & $\frac{n}{i n}$ & 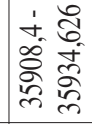 & 站 & & 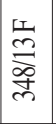 & $\stackrel{m}{m}$ & 衣 & 量 & 产 & 资 & $\begin{array}{l}\text { 营 } \\
\text { d }\end{array}$ & 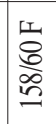 & 訔 & \\
\hline 3 & 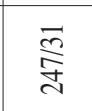 & 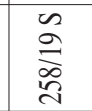 & 옹 & 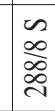 & 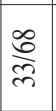 & $\frac{\infty}{\frac{\infty}{q}}$ & 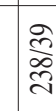 & 号 & $\begin{array}{l}\text { 売 } \\
\text { 严 }\end{array}$ & $\frac{n}{\frac{n}{g}}$ & 흠 & 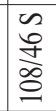 & 赵 & 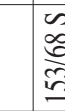 \\
\hline$\approx$ & 葶 & 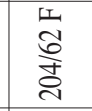 & 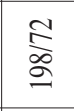 & : & 字 & 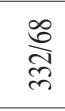 & 胥 & 竞 & 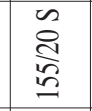 & ते & $\begin{array}{l}\text { 訔 } \\
\stackrel{\Xi}{\Xi}\end{array}$ & 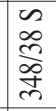 & 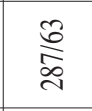 & \\
\hline$=$ & $\underline{\underline{5}}$ & 㕝 & 흠 & 兽 & \& & 일 & 㐫 & ปิ & 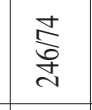 & 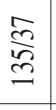 & $\frac{n}{\stackrel{n}{\circ}}$ & $\begin{array}{l}\text { 总 } \\
\text { s. }\end{array}$ & 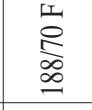 & \\
\hline 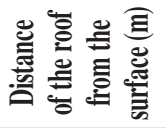 & $\pi$ & $\ddot{\infty}$ & 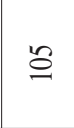 & $\stackrel{\varrho}{\varrho}$ & $\stackrel{\infty}{\stackrel{\infty}{2}}$ & $\Xi$ & ำ & 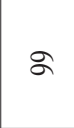 & $\approx$ & $\underset{\sim}{2}$ & त & $\cong$ & $\infty$ & \\
\hline 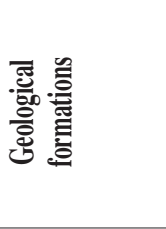 & 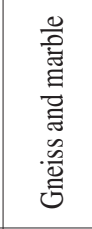 & 惫 & 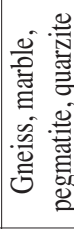 & & 言 & डั & 彦 & 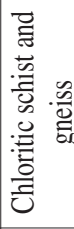 & 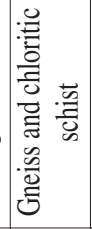 & 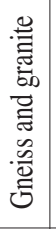 & 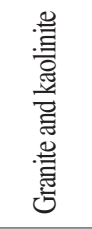 & 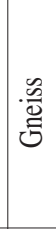 & 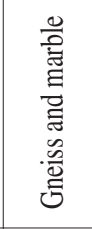 & है \\
\hline 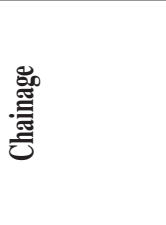 & 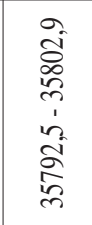 & 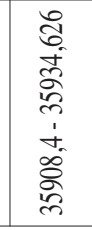 & 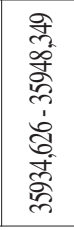 & $\bar{m}$ & & 串 & 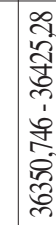 & 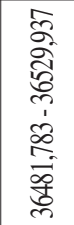 & 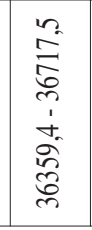 & 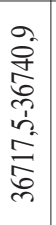 & 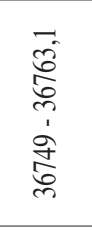 & 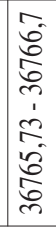 & 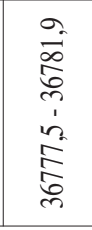 & $\infty$ \\
\hline
\end{tabular}



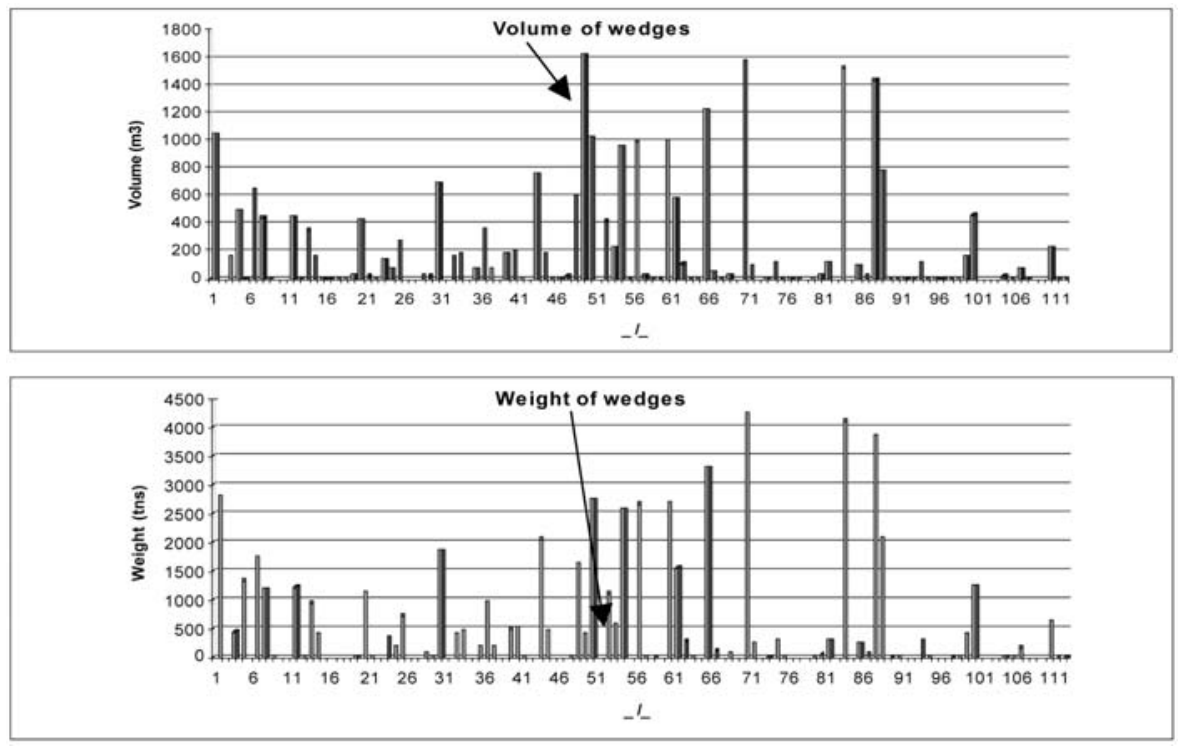

Fig. 2: Comparison between volume and weight of wedges. The arrow shows the position of wedge with volume of $1646,741 \mathrm{~m}^{3}$, and weight of 446,2 tns.

\section{Excavation methods}

The rock mass along the tunnel differs from one place to another. Hard gneiss rock fair qualified of marble of granite was alternated with fractured and deformated rock mass of gneiss and marble. Furthermore, the presence of chloritic schist and the shear zone, minimize the safety of the excavation using the simple mechanical means. So, in order to excavate the tunnel safety, we ought to apply different excavated methods, taking into account rock mass behaviour (Marinos et al, 2005).

Near the outlets and where the rock mass was very poor, the tunnel was excavated mechanically, using the NATM method of excavation. The use of explosive measures was preferred on poor and fair quality of hard rock mass. The excavation of chloritic schist and the shear zone was very dangerous. Although the chloritic schist was very hard and it was very difficult to be excavated with mechanical means, it was deformated very quickly, when it was in conduct with the atmosphere. So, before the removal of excavation material to be completed, pieces of chloritic schist were felled down. The SCL method of excavation was preferred on that case in order to support small parts of the face before the excavation be completed. Furthermore, light explosion used in order to crack the hard rock mass helping the excavation.The sudden change of rock mass quality created the necessity of fore polling.

\section{Tunnel stability}

The sliding along a plane, the décollement from the roof and the fall of wedges (Chatziangelou et all, 2001) were the common failure causes. Sliding took place along a tectonic surface from the walls of the tunnel. On the other hand, the dècollement of a plate is due to its smooth surface in addition with the influence of gravity (Table 1).

One hundred and eleven wedges were measured along the tunnel (Table 2). All the wedges were to be collapsed, so the calculated safety factor, before the application of support was zero. From ch.36+139,41 to ch.36+176,222 a wedge with volume of $19244,17 \mathrm{~m}^{3}$ had been observed on the 

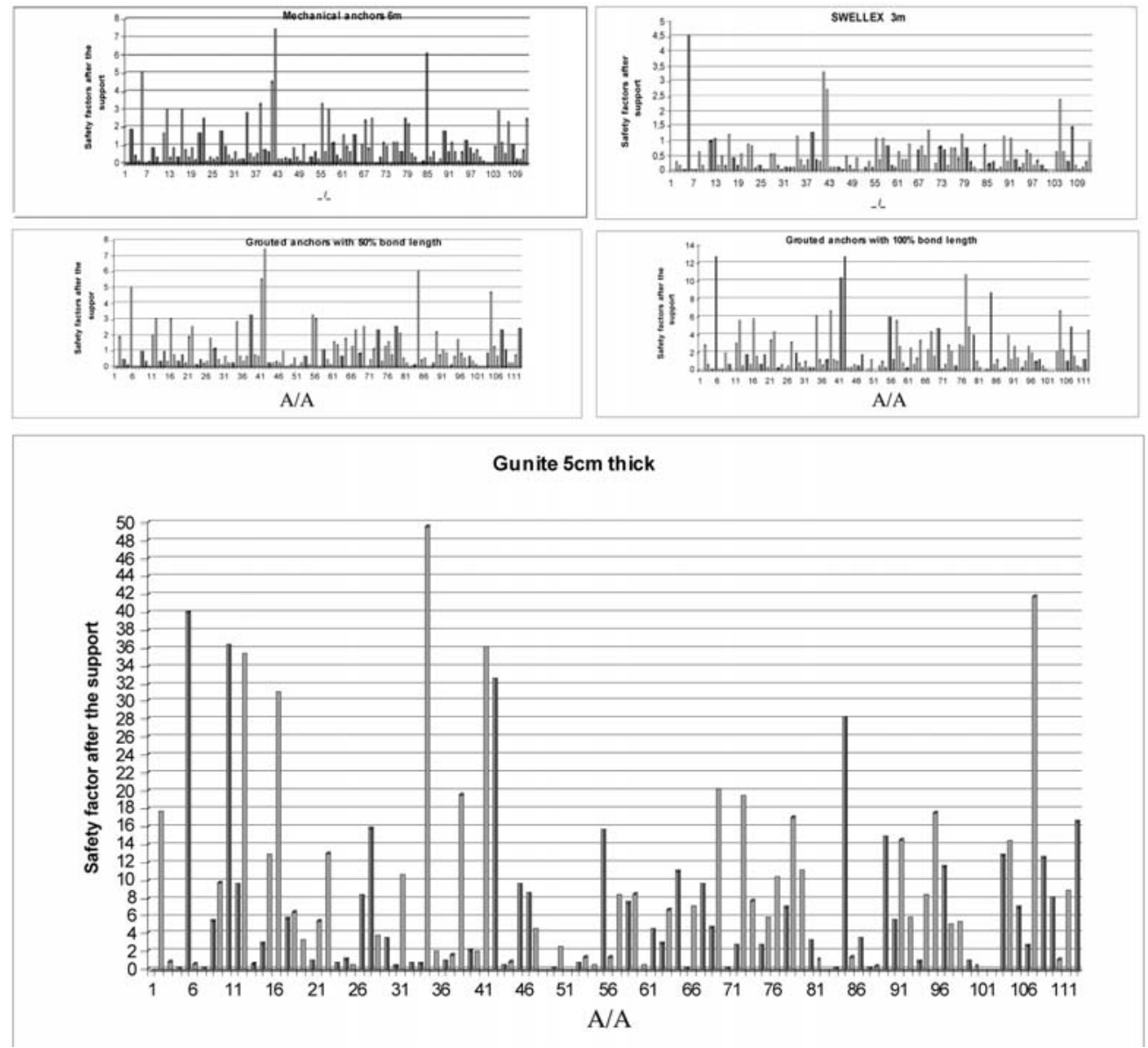

Fig. 3: Safety factors of the wedges after the support of different measures.

upper right part of the tunnel. The failure of that wedge could cause the collapse or all the overlying formations up to the surface. That wedge didn't take into account on our estimations. Another one big wedge, with volume of $4390,22 \mathrm{~m}^{3}$ (from ch.36+215.595 to ch.36+240,379), which was also formed on the upper right part of the tunnel didn't take into account on our estimations.

Usually, there is a relation between the weight and the volume of the wedges. It is common place, the wedges with big volume to be also heavy. But an exception of the above, was observed between ch.35+710 and ch.35+716,5, where there is a wedge with the one of the biggest volumes $\left(1646,741 \mathrm{~m}^{3}\right)$, but one of the slightest ones (weighted 446,2 tns) (Fig.2) That is due to the very poor quality of the rock mass, in addition to fractrure and deformation. The deformation reduced the apparent weight of the rock mass. Also, the numerous of discontinuities, as they are crossed, they cause empty space at the cross point, so the weight of the wedge does not increase so much as the volume increase.

\section{Comparing different support measures}

The rock mass quality methods, RMR and GSI, were used for determining the efficacious support measures of the slopes and the tunnels in the area (Christaras et al, 2002). According to the geotechnical 


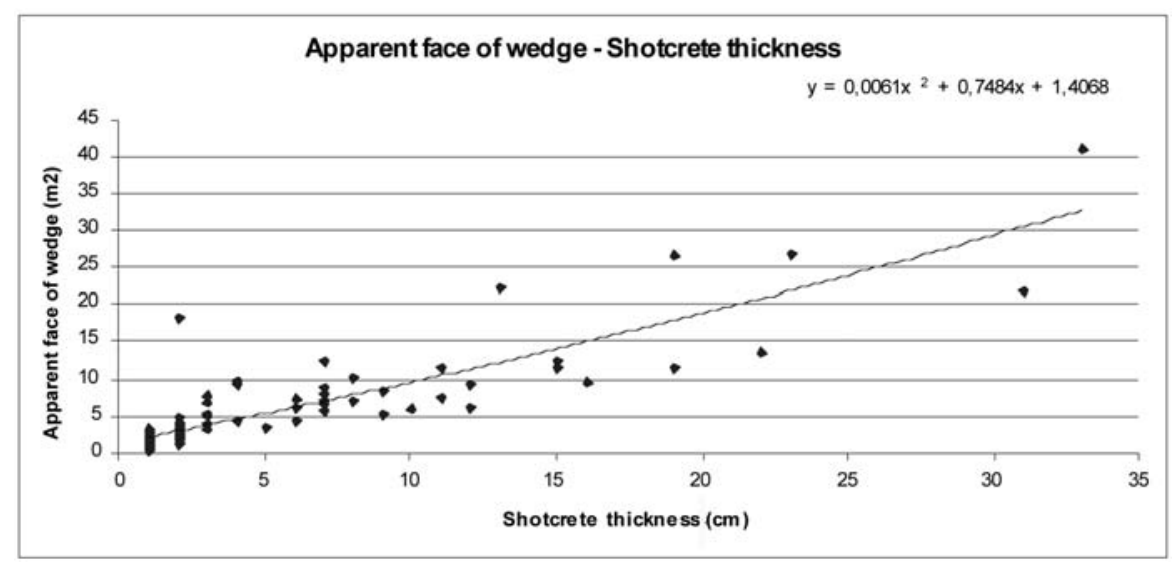

Fig. 4: Relationship between apparent face of wedge and shotcrete thickness.

characteristics of the rock mass, the proposed support measures are completed with different types. The present paper examines the effectiveness of different types of anchors and shotcrete on the rock mass of Symbol Unit. For this purpose, the support of the tunnel was tested using mechanical anchors $6 \mathrm{~m}$ long, swellex $3 \mathrm{~m}$ long, grouted anchors $3 \mathrm{~m}$ long with $50 \%$ bond length, grouted anchors $3 \mathrm{~m}$ long with $100 \%$ bond length and shotcrete with thickness of $5 \mathrm{~cm}$ (Fig.3). Actually, the wedges tested to be supported with one of the above measures, without using combination of them. The required safety factor which was used for the comparisons was 1,5 .

Twenty five wedges were observed to be supported with mechanical anchors with length of $6 \mathrm{~m}$. Five wedges were supported with swellex bolts. So, the mechanical anchors can support more wedges than the swellex bolts can. Also, there is no difference when the bolts are grouted at $50 \%$ of their length and are totally not grouted. The safety becomes bigger when the bolts are totally grouted. Forty seven wedges are supported sufficiently. Also, the grouted anchors with $100 \%$ bond length give bigger safety factors than the grouted anchors with $50 \%$ bond length. The percent of safety increases two times with the use of grouted anchors with $100 \%$ bond length. As far as shotcrete concern, seventy four wedges are supported effectively with shotcrete $5 \mathrm{~cm}$ thick.

\section{Calculation of shotcrete thickness using the apparent face of wedge}

As the excavation of tunnels and the application of the support measures are dangerous, the quick calculation of shotcrete thickness during the excavation is useful. Comparing the apparent face of the wedges (the surface which is appeared at the inner surface of the tunnel) with the demanded shotcrete thickness (thinner than $40 \mathrm{~cm}$ ), in order the unstable wedges to be supported, a relationship was resulted (Fig.4);

$$
\mathrm{F}\left(\mathrm{m}^{2}\right)=0,0061 *[\mathrm{~h}(\mathrm{~cm})]^{2}+0,7484 * \mathrm{~h}(\mathrm{~cm})+1,4068
$$

where $\mathrm{h}=$ shotcrete thickness $(\mathrm{cm})$

$\mathrm{F}=$ apparent face of the wedge $\left(\mathrm{m}^{2}\right)$

The coefficient of the above relationship was calculated 0,877 .

The above relationship has the same form with the relationship, which has calculated from the data of Asprovalta tunnels of Egnatia Highway (Chatziangelou, 2008);

$$
\mathrm{F}\left(\mathrm{m}^{2}\right)=0,3489 *[\mathrm{~h}(\mathrm{~cm})]^{2}+16,654 * \mathrm{~h}(\mathrm{~cm})+14,049
$$


Asprovalta tunnels are located at Serbomakedonian mass and the tunnels are passed through gneiss with pegmatitic intercalations, marble and amphibolite. The coefficient of that relationship was calculated 0,082 .

\section{Conclusions-Results}

The tunnel which crosses the Symbol Mountain was excavated dangerously because of the difficult geological status with unexpected failure conditions. The sliding along a plane, the décollement from the roof and the fall of wedges were the common failure causes.

Different methods were used in order to excavate the tunnel safely. The NATM method of excavation was used near the outlets and where the rock mass was very poor. On poor and fair quality of hard rock mass the explosive measures were the most effective. Also, light explosion was used in order to crack the hard rock mass helping the excavation. Chloritic schist formation and the places, where the loose deformed material flowed from the walls and the face, were excavated by the SCL method.

Studying the geometrical characteristics of wedges, we concluded that the weight reduce of the wedges with big volume is due to i) deformation which reduces the apparent weight of the rock mass and ii) the cross of the numerous discontinuities, that they cause empty space at the cross point. Examining the effectiveness of different types of anchors and shotcrete, we concluded that the mechanical anchors can support more wedges than the swellex bolts can. Also, there is no difference when the bolts are grouted at $50 \%$ of their length and are totally not grouted. The safety becomes bigger when the bolts are totally grouted. As far as shotcrete concern, more than $50 \%$ of wedges are supported effectively with shotcrete $5 \mathrm{~cm}$ thick.

Finally, comparing the apparent face of the wedges with the demanded shotcrete thickness (thinner than $40 \mathrm{~cm}$ ), in order the unsteady wedges be supported, a relationship (1) was resulted. The above relationship has the same form with the relationship (2), which has calculated from the data of Asprovalta tunnels of Egnatia Highway;

Consequently, there is a relation between apparent face of the wedges and the demanded shotcrete thickness being formed;

$$
y=a * x^{2}+b * x+c
$$

\section{References}

Bieniawski, Z.T., 1989. Engineering rock mass classifications. New York: Wiley.

Chatziangelou M., Christaras B., Dimopoulos G., Soulios G., Kilias A., 2001. Support of unstable wedges along the Platamon railway tunnel under construction, in northern Greece. Journ. Eng. Geology, Elsevier, Amsterdam, Ed.nr.1060.

Chatziangelou M., 2008. Estimation of failure conditions that appears on poor quality rock mass of Asprovalta tunnels and support measures, P.H.D. Thesis, Aristotle University of Thessaloniki .

Christaras, B., Chatziangelou, M., Malliaroudakis, Em.\& Merkos, S., 2002. Support Capacity of wedges and RMR classification along the Asprovalta tunnel of Egnatia Highway, in n. Greece, $9^{\text {th }}$ Congress of the International Association for Engineering Geology and the Environment, J.L. van Rooy and C.A. Jermy, ISBN No.0-620-28559-1.

Hoek, E., 2004. Rockmass classification. Hoek's Corner < www.rockscience.com > (accessed December 2004)

Marinos, P., Hoek, E., Kazilis, N., Agistalis, G. \& Marinos, V., 2005. The tunnels of Egnatia highway, Greece. Design and construction in a variety of rock masses under difficult geological conditions. Geology and linear infractures. Proccedings of Int. Symposium, Lion, 2005. 